\title{
BMJ Open A protocol for a Canadian prospective observational study of decision-making on active surveillance or surgery for low-risk papillary thyroid cancer
}

\author{
Anna M Sawka, ${ }^{1}$ Sangeet Ghai, ${ }^{2}$ George Tomlinson, ${ }^{3}$ Lorne Rotstein, ${ }^{4}$ \\ Ralph Gilbert, ${ }^{5}$ Patrick Gullane, ${ }^{5}$ Jesse Pasternak, ${ }^{4}$ Dale Brown, ${ }^{5}$ John de Almeida, ${ }^{5}$ \\ Jonathan Irish, ${ }^{5}$ Douglas Chepeha, ${ }^{5}$ Kevin Higgins, ${ }^{6}$ Eric Monteiro, ${ }^{7}$ \\ Jennifer M Jones, ${ }^{8}$ Amiram Gafni, ${ }^{9}$ David P Goldstein ${ }^{5}$
}

To cite: Sawka AM, Ghai S, Tomlinson G, et al. A protocol for a Canadian prospective observational study of decisionmaking on active surveillance or surgery for low-risk papillary thyroid cancer. BMJ Open 2018;8:e020298. doi:10.1136/ bmjopen-2017-020298

- Prepublication history for this paper is available online. To view these files, please visit the journal online (http://dx.doi. org/10.1136/bmjopen-2017020298).

Received 25 October 2017 Revised 12 January 2018 Accepted 22 February 2018

Check for updates

For numbered affiliations see end of article.

Correspondence to

Dr Anna M Sawka;

sawkaam@yahoo.com

\section{ABSTRACT}

Introduction Low-risk papillary thyroid cancer (PTC) is increasingly being diagnosed throughout the world; yet the mortality risk is low compared with other malignancies. Traditional management includes thyroid surgery, sometimes followed by radioactive iodine and thyroid hormone treatment. Active surveillance (AS) has been proposed as a means to reduce overtreatment of PTC. AS involves close disease follow-up, with the intention to intervene if the disease progresses, or on patient request. Methods and analysis This is a multiphase prospective observational study. In the first phase of this study, consenting eligible adults with low-risk PTC, that is, $<2 \mathrm{~cm}$ in maximal diameter, confined to the thyroid and not immediately adjacent to critical structures in the neck, are provided verbal and written information about PTC disease prognosis following surgery or AS. Questionnaires are administered at baseline and after the disease management decision on AS or surgery is finalised. Patients may choose either option (surgery or $\mathrm{AS}$ ), and the primary outcome is the frequency with which either disease management option is chosen. Secondary outcomes include: rationale for the decision, role of the patient in decision-making and decision satisfaction. In the second phase of the study, consenting eligible adult patients who completed the first study phase may enrol in respective AS or surgery group follow-up studies. The following outcomes are examined 1 year after enrolment in the follow-up phase: decision regret about disease management choice (primary outcome), psychological distress, disease-specific quality of life, fear of disease progression, body image satisfaction, disease progression, crossover to surgery in the AS group, new chronic thyroid hormone use and healthcare resource utilisation.

Ethics and dissemination The University Health Network Research Ethics Board approved this study (ID 15-8942). The results will be published in an open access journal. Trial registration number NCT03271892; Pre-results.

\section{INTRODUCTION}

Increased utilisation of diagnostic imaging has resulted in increased diagnosis of

\section{Strengths and limitations of this study}

- A strength of this study is that it is the first study to conduct a detailed prospective evaluation of decision-making related to the choice of active surveillance (AS) or immediate thyroid surgery, for management of low-risk papillary thyroid cancer (PTC).

- Another strength of this study is the inclusion of secondary outcomes such as: psychological distress (depression and anxiety), disease-specific quality of life, fear of disease progression and body image satisfaction.

- A limitation of this study is that participants are not randomised to AS or surgical arms, as the choice of disease management is per the patient and treating physician.

- Another limitation of this study is that for patients who undergo thyroid cancer surgery, the surgical procedure and associated follow-up procedures are at the discretion of the treating physician and patient, which is intended to be reflective of usual care.

incidental malignancies that have a low risk of progression or death. ${ }^{1}$ Some recent annual estimates on the number of individuals newly diagnosed with thyroid cancer include: 298000 worldwide, ${ }^{2} 7100$ in Canada, ${ }^{3} 56870$ in the USA, ${ }^{4} 53000$ in Europe $^{5}$ and 3400 in the UK. ${ }^{2}$ In Canada, thyroid cancer incidence is rising faster than any other malignancy, ${ }^{3}$ with an increase of $156 \%$ from 1991 to $2006^{6}$. The survival rate of thyroid cancer is among the highest of all malignancies ${ }^{34}$ and the 5-year survival rate for early stage (local) thyroid cancer is reported to be $>99 \%$. Most of the increase in thyroid cancer incidence is attributed to detection of papillary thyroid cancer (PTC) $\leq 2 \mathrm{~cm}$ in diameter, ${ }^{7-11}$ especially localised disease without metastases (ie, no spread of disease beyond the 
thyroid) ${ }^{81112}$ PTCs $\leq 2 \mathrm{~cm}$ in diameter are estimated to account for $60 \%-68 \%$ of new cases. ${ }^{79}$ Thyroid cancer treatment traditionally involves thyroid surgery, which may be followed by thyroid hormone replacement (lifelong) and radioactive iodine treatment. ${ }^{13-15}$ However, treatment-related morbidity in individuals at low risk of dying from their disease is a relevant concern; thus, the consideration of an option for a conservative, non-operative management approach for select to low-risk PTC cases has been proposed by some experts. ${ }^{16-18}$

Active surveillance (AS) of a malignancy consists of close clinical/diagnostic test follow-up (in lieu of immediate surgery), with the intention of treatment with curative intent if the disease progresses or the patient requests it. In two recent prospective observational studies from Japan, patients with PTC $\leq 1 \mathrm{~cm}$ in diameter underwent AS and there were no PTC-related deaths and no distant metastatic recurrences, and the rate of cervical lymph node recurrence was approximately $1 \%$ to $2 \%$ (followed on average for 5-6 years). ${ }^{19}{ }^{20}$ Furthermore, primary tumours under AS did not significantly grow (ie, $\geq 3 \mathrm{~mm}$ diameter increase) in $93 \%$ to $95 \%$ of individuals over this time period. ${ }^{19}{ }^{20}$ Moreover, all cases of disease progression under AS were successfully cured with surgery ${ }^{19} 20$ and the majority of patients who initially accepted AS avoided thyroid surgery $(84 \%-94 \%$ over about 5-6 years). ${ }^{19}{ }^{20}$ Furthermore, in a recently published report from an ongoing American AS study, Tuttle et al reported low rates of tumour growth $(3.8 \%$ of cases) and no evidence of incident metastatic disease, in a cohort of 291 patients with PTC whose primary tumour was $\leq 1.5 \mathrm{~cm}$ in maximal diameter (median 25-month follow-up period) ${ }^{21}$

A highly important consideration is the extent to which AS may be considered as an acceptable disease management by patients diagnosed with low-risk PTC. In the largest study of AS in Japan, $55 \%$ of individuals with papillary microcarcinoma chose to undergo AS, when offered this option or surgery. ${ }^{22}$ Similar data are not yet available from other parts of the world. However, the recent report of 291 patients with low-risk PTC that have been enrolled in an AS study in the USA ${ }^{21}$ suggests that there may be interest in this option in North America. In an in-depth qualitative study from the same institution, the rationale for patient choice of surgery or AS was studied in 15 patients. ${ }^{23}$ In this study, D'Agostino et al reported that patients who opted for surgery perceived a strong threat of the disease and were motivated to cure the malignancy, whereas those who chose AS perceived the disease to be relatively indolent and were motivated to avoid living without their thyroid (and possible reliance on thyroid hormone replacement) ${ }^{23}$ The frequency with which Canadian with low-risk PTC would prefer AS or surgery (and the rationale for the choice) is unknown. Furthermore, prospectively collected quantitative data on decision-making process and relevant psychosocial/ quality-of-life patient-reported outcomes are needed for patients with low-risk PTC offered the options of surgery or AS.
DESIGN, METHODS AND ANALYSIS

\section{Study design and setting}

We are conducting a multiphase prospective observational study of patients with low-risk PTC. The study is currently being conducted at the University Health Network (UHN) hospitals in Toronto (including Toronto General Hospital, Princess Margaret Cancer Centre and Toronto Western Hospital), with the plan to add additional sites, if feasible.

Study aim and primary outcomes in respective study phases In the first phase of this study, our aim is to prospectively examine the decision-making process of patients with low-risk PTC considering surgery or active surveillance and the primary outcome is the frequency (percentage) of patients choosing AS or surgery, respectively. In the second phase of the study, consenting patients who completed the first phase of the study are followed in the respective study arms of (1) active surveillance or (2) surgery (according to patient choice). The primary outcome in the second phase of the study is decision regret (with respect to the decision on AS or surgery, to be described in respective arms).

\section{The study population eligibility criteria and recruitment}

In the first phase of the study, we are enrolling consenting eligible adults (age $\geq 18$ years) with surgically untreated low-risk PTC that is confined to the thyroid, not immediately adjacent to critical structures in the neck (eg, trachea or recurrent laryngeal nerve), and measures $<2 \mathrm{~cm}$ in maximal diameter (table 1). The inclusion criteria, relating to the primary tumour characteristics, were reviewed and approved (by consensus) by all thyroid cancer surgeons in our institution. In the first study phase, study participants are provided verbal and written information about thyroid cancer disease prognosis and information about AS. This information is regularly updated to reflect the evolving evidence on long-term outcomes with AS of low-risk PTC. Participants are free to choose either surgery or AS for management of their thyroid cancer. Eligible consenting patients who have completed the first phase of this study (and rendered a disease management decision) may enrol in the second phase of the study, which includes study follow-up of respective disease management arms of: (1) active surveillance or (2) thyroid cancer surgery. Patient recruitment is focused in participating thyroid cancer surgical clinics, although eligible patients may be referred by other healthcare providers or self-referred. All patients received a formal consultation from a thyroid cancer surgeon (of their choice) prior to consideration of enrolment in any phase of the study. Patients who may be eligible for the study are offered the opportunity to meet with a research assistant. Screening for eligibility is performed by a research assistant, under the supervision of one or more of the primary coprimary investigators (DPG, AMS). Baseline neck imaging studies are reviewed by a study radiologist (SG) and surgeon (DPG) to confirm eligibility. 
Table 1 Details of study inclusion and exclusion criteria (At baseline assessment, prior to deciding on surgery or active surveillance) $)^{\star}$

\section{Inclusion criteria}

Age $\geq 18$ years

\section{Exclusion criteria}

Known regional or distant metastatic thyroid cancer at the time of baseline evaluation (prior to thyroid cancer surgery)

Newly diagnosed, previously untreated papillary thyroid cancer A history of prior thyroid cancer surgery

(PTC) $<2 \mathrm{~cm}$ in maximal diameter on ultrasound imaging.

Fine needle aspiration biopsy of the primary tumour must be

read as either PTC or suspicious for PTC (as reviewed by a

cytopathologist at a participating study site).

No evidence of metastatic cervical lymphadenopathy on ultrasound imaging of the neck (or other neck imaging).

No other potential indication for thyroid or parathyroid surgery at the time of the assessment.

Patient permission must be granted for review of thyroid cancer-related medical records to determine study eligibility
The primary PTC is adjacent to the recurrent laryngeal nerve or trachea

Known or suspected poorly differentiated or non-papillary thyroid cancer

Medically unfit for surgery due to comorbidity

Another active malignancy (excluding non-melanoma skin cancer) for which patients are receiving treatment or are less than 3 years from completing treatment.

Pregnancy at the time of study enrolment

Other current indications for thyroid or parathyroid surgery

Patient is unable to provide informed consent for the study or comply with study follow-up procedures due to current severe active cognitive or psychiatric impairment, substance abuse or other reasons.

\begin{abstract}
*Eligible consenting patients participating in the respective follow-up arms of the study (ie, active surveillance or surgery) must have been enrolled in the first phase of the study, where standardised information about papillary thyroid cancer prognosis and active surveillance is offered. Consenting eligible patients in the surgical follow-up arm are enrolled after first thyroid cancer surgery is completed.
\end{abstract}

\section{Study follow-up assessments and outcomes}

In the first phase of the study (decision-making phase), a baseline medical history, physical examination and laryngoscopy (if not already performed) are performed. Several questionnaires are administered at baseline (prior to the presentation of the information about AS), as well as after the disease management decision on AS or surgery is finalised (generally within a few months of study enrolment). Baseline questionnaires include questions on demographic and medical history, coping mechanisms (Brief Cope Questionnaire ${ }^{24}$ ), fear of disease progression (Short form of the Fear of Progression Questionnaire ${ }^{2526}$ ), fear of surgery (Surgical Fear Questionnaire ${ }^{27}$ ) and decision self-efficacy (Decision Self-Efficacy Scale $\left.^{28}\right)$. The primary outcome is the frequency with which either disease management option is chosen by the patient (AS or immediate surgery). Secondary outcomes include: rationale for the decision, role of the patient in decision-making and decision satisfaction. ${ }^{29}$

In the second phase of the study, consenting eligible adult patients who completed the first study phase may enrol in a follow-up study of respective AS or surgery arms. The active surveillance arm includes follow-up assessments by study investigators at least every 6 months for 2 years, followed by yearly (if no evidence of disease progression). These assessments include clinical history and examination, neck ultrasound and measurement of thyroid-stimulating hormone, free thyroxine, thyroglobulin and thyroglobulin antibody. More frequent assessments or additional investigations may be arranged, depending on clinical circumstances. Thyroid hormone treatment is offered as per current clinical practice guidelines for chronic management of low-risk PTC, ${ }^{13}$ but its use is not mandated for participation in the AS follow-up study. The criteria for disease progression prompting

Box 1 Definition of progression of papillary thyroid cancer under active surveillance for which salvage surgery is advised (one or more of the criteria listed below)*

- Primary index papillary thyroid cancer (PTC) growth $>3 \mathrm{~mm}$, confirmed on two consecutive ultrasound examinations. The $3 \mathrm{~mm}$ size cut-off has been shown to be safe and effectively treated in prior PTC active surveillance studies. ${ }^{19} 2022$

- Primary PTC growth in a location that is concerning (eg, immediately adjacent to the trachea or in the course of the recurrent laryngeal nerve).

- Incident development of metastatic PTC to lymph nodes (confirmed on cytology or unequivocal imaging)

- Incident development of distant metastatic PTC (confirmed on imaging or biopsy or surgical histology)

*Patients may choose to have surgery in the absence of disease progression at any time point in follow-up. 
a recommendation for surgery are shown in box 1 . All patients under active surveillance are free to choose to have surgery at any time point, in absence of disease progression. For consenting patients who choose surgery for primary management of their disease, the treating surgeon and patient will decide on the extent of the surgery and associated clinical follow-up. For study participants in either arm that undergo thyroid cancer surgery, there are no restrictions on which surgeon or where the surgery may be performed (ie, as per patient choice).

One year after enrolment in the respective AS or surgical follow-up study arms, the following study outcomes are examined: decision regret about disease management choice (primary outcome) (Decision Regret Scale $^{30}$ ), psychological distress (depression and anxiety, measured by the Hospital Depression and Anxiety Scale $^{31}$ ), disease-specific quality of life (measured by the thyroid cancer module of the MD Anderson Symptom Inventory ${ }^{32}$ ), fear of disease progression ${ }^{25} 26$ and body image satisfaction (measured by the Body Image Scale ${ }^{33}$ ). Furthermore, thyroid cancer-related medical records are examined in all patients to evaluate for disease progression, crossover to surgery in the AS group, new chronic thyroid hormone use and thyroid cancer-related healthcare resource utilisation. Consent for review of medical records is requested for a minimum of 3 years, but an optional consent is requested for review of records up to 10 years. However, indefinite clinical follow-up is offered for patients under AS, who do not undergo surgery.

\section{STATISTICAL CONSIDERATIONS}

\section{Sample size calculation}

There is no a priori calculated sample size for the first phase of the study (on medical decision-making), as it is a descriptive study incorporating a convenience sample; however, the study will continue enrolling patients to adequately power meaningful analysis of the primary outcome in the follow-up study. As our primary analysis for the second phase of the study is a description of the level of decision regret in the respective AS and surgical arms at 1 year, a convenience sample should technically suffice. However, we are planning a secondary analysis, comparing decision regret between the AS and surgical groups, assuming sufficient sample size, so a sample size justification for that analysis is herein provided. There is no published data on what difference in level of regret is considered unimportant, so we chose our noninferiority boundary based on the following considerations. Pilot decision regret data collected on a low-risk PTC sample of 74 patients in a treatment decision-making showed a between-patient SD of 16 points (Sawka, unpublished data). Norman reported that across a wide range of questionnaires, the minimally important difference (MID) was around one-half an SD. ${ }^{34}$ The non-inferiority boundary should be smaller than the MID, as it represents an unimportant difference. We select a value of $0.375 \mathrm{SD}$ or six points. The decision regret scale sums scores on five questions, each scored $1-5$ and transforms to a $0-100$ range. ${ }^{30}$ This means that a difference of one level on one question corresponds to five points on the final scale. Our non-inferiority boundary allows an average difference of 1 level on one question, but little more. The level of decision regret in the AS group will be considered non-inferior if the upper end of the $95 \%$ one-sided interval for the difference in mean regret scores lies below the value of the non-inferiority boundary. The minimum sample size required to demonstrate that decision regret which is not inferior at 1 year in patients choosing AS compared with those choosing thyroid surgery is a total of 180 patients from the combined study arms (assuming $80 \%$ power, a one-sided 95\% CI and an MID of six points on the decision regret questionnaire). As there may be some attrition during the study, we will target a combined sample size from the follow-up arms of approximately 200 patients. The enrolment in the first phase of the study (on the choice of AS or surgery) will stop once 200 patients have been recruited in the combined AS and surgical arms of the follow-up study. We cannot control the number of patients enrolling in either arm, as the ultimate treatment decision is based on patient choice; thus, our recruitment target is based on the total number of patients in both follow-up study arms.

\section{Statistical analysis}

The first phase of the study (AS or surgery decision-making) is a descriptive study, and the primary outcome of percentage (and 95\% CI) of study participants who ultimately choose AS or surgery. Demographic and disease characteristics will be descriptively summarised, with means and SD for continuous outcome and number and percentages for categorical outcomes. All baseline questionnaire data will be scored as per developers, for total scores or subscale scores and results summarised for the entire study population (as well as the ultimate disease management subgroups). If there is a sufficient number of patients in both AS and surgical groups, then we will compare the baseline characteristics between groups, using unpaired Student's t-tests. Furthermore, if there is a sufficient number of patients for analysis, a predictive analysis examining predictors of choosing AS will be performed using a logistic regression analysis (incorporating demographic and disease factors). We will use a previously reported concurrent mixed methods approach $^{35} 36$ to ascertain patients' reasons for treatment choice, by collecting data from semistructured questions, coding responses and identifying themes.

In the follow-up phase of the study, the primary analysis will be a descriptive analysis of the level of decision regret, ${ }^{30}$ expressed as a mean and SD in the AS arm and surgical arm, respectively. Assuming a sufficient number of participants in both the AS and surgical arm for meaningful analysis, an unpaired Student's t-test will be performed, comparing decision regret scores in each group. The comparative analysis of decision regret scores between groups will be a non-inferiority (one-sided) comparison. 
Other quantitative data from all other questionnaires for each treatment subgroup will be summarised descriptively, as means and $95 \% \mathrm{CI}$, as appropriate for the questionnaire scores and subscale scores.

\section{ETHICS AND DISSEMINATION Informed consent}

Informed consent is obtained from study participants enrolling in the respective parts of the study, including (1) decision-making on disease management (AS or surgery) and (2) (a) active surveillance follow-up arm, (b) surgical arm. Patients are assured that their participation is voluntary and they may withdraw from the study at any time. Furthermore, for patients withdrawing from the study, assistance with arrangement of continuity of clinical follow-up care will be offered, if needed. Furthermore, patients opting for AS may change their minds and have thyroid surgery at any point in follow-up (regardless of whether the disease has progressed or not). Participants may choose to provide optional consent for additional follow-up up to 10 years for any aspect of the study.

\section{Study registration, ethics review and data protection}

This study is registered at Clinicaltrials.gov: NCT03271892. Research ethics board approval for the study has been obtained. If additional study sites are added, additional research ethics board approval will be obtained at all participating sites and UHN.

\section{Access to data and dissemination}

Only the study coprimary investigators (AMS, DPG), research staff and statistician will have access to the raw data, which will be securely stored. All participants are assigned a unique study identifier number. No identifying patient information will be shared. Aggregate study results will be presented at scientific conferences and the results of the study published in one or more peer-reviewed open access journals. Study results will also be disseminated to local thyroid cancer specialists and patient support group representatives.

\section{Recruitment and status of the study}

This study is currently approved by the UHN Research Ethics Board and enrolment is in progress. The total duration of the study is expected to be up to 10 years and further research funding will be sought for support of longterm follow-up and outcome assessment. An important limitation of our study is that we do not have preliminary pilot data on the feasibility of recruitment of Canadian low-risk thyroid cancer patients in the active surveillance arm. However, at the current recruitment rate, it appears that our recruitment target will be achieved within the 10 -year time frame of the study.

\section{Perspective}

Management of low-risk PTC is currently evolving, with a trend for providing more conservative options for patients who are at lowest risk of dying of their disease. ${ }^{13}$
AS has been proposed as a means to mitigate potential overtreatment and treatment-related complications in patients with low-risk PTC. ${ }^{16-18}$ Furthermore, in Japan, AS has emerged as a viable treatment option for papillary microcarcinoma. ${ }^{19}{ }^{20}$ Yet, outside of Japan, the acceptability of AS among patients with low-risk PTC is unknown, and more research is needed examining the long-term outcomes (clinical and psychosocial) in patients with larger tumour sizes. This prospective study is intended to complement that of existing research in AS of PTC from other parts of the world, and inform potential expansion of disease management options for future patients with low-risk PTC. It is important to note that long-term follow-up of patients enrolled in this, and other studies, examining active surveillance of low risk PTC is needed.

\section{Author affiliations}

${ }^{1}$ Department of Endocrinology, University Health Network, University of Toronto, Toronto, Ontario, Canada

${ }^{2}$ Department of Radiology, University Health Network, University of Toronto, Toronto, Ontario, Canada

${ }^{3}$ Department of Epidemiology and Biostatistics, University Health Network, University of Toronto, Toronto, Ontario, Canada

${ }^{4}$ Department of Surgery, University Health Network, University of Toronto, Toronto, Ontario, Canada

${ }^{5}$ Department of Otolaryngology, Head and Neck Surgery, University Health Network, University of Toronto, Toronto, Ontario, Canada

${ }^{6}$ Department of Otolaryngology, Sunnybrook Health Sciences Centre, University of Toronto, Toronto, Ontario, Canada

${ }^{7}$ Department of Otolaryngology, Mount Sinai Hospital, University of Toronto, Toronto, Ontario, Canada

${ }^{8}$ Department of Psychosocial Oncology, University Health Network, University of Toronto, Toronto, Ontario, Canada

${ }^{9}$ Centre for Health Economics and Policy Analysis, McMaster University (HSC-3H29), Hamilton, Ontario, Canada

Acknowledgements The authors are grateful to Dr Akira Miyauchi and Dr Michael Tuttle, who answered questions and provided advice on the design of the AS clinical protocol. The authors would also like to acknowledge the valuable assistance of the study research staff, including Tom Yoannidis, Coreen Marino and Jeverlynn Danzie.

Contributors The coprimary investigators, DPG and AMS designed the study, obtained funding for the study, oversaw all aspects of execution and reporting of the study. SG has provided input in study design and is the primary study radiologist, providing input on interpretation of ultrasound imaging of the neck. All of the surgeon investigators have provided input in study design, are active in assisting in participant recruitment for the study and provided input on this manuscript (LR, RG, PG, JP, DB, JdA, JI, DC, KH, EM). GT, JMJ and AG have provided input in methodological aspects of the study design and assisted in the application for study funding. GT is the statistician overseeing analysis of the study results. All authors have approved the final manuscript.

Funding Funding for the first phase of this study (medical decision-making) was obtained from an operating grant (Innovation Grant) from the Ontario Academic Health Sciences Centres Alternate Funding Plan Innovation Fund (Ontario Ministry of Health). The follow-up phase of the study (AMS and surgical arm follow-up to 1 year) is funded by an operating grant from the Canadian Cancer Society Research Institute (The Lotte and John Hecht Memorial Foundation Innovation Grant, \#703948).

Competing interests None declared.

Patient consent Obtained.

Ethics approval This study is approved by the University Health Network Research Ethics Board in Toronto, Canada.

Provenance and peer review Not commissioned; externally peer reviewed.

Open Access This is an Open Access article distributed in accordance with the Creative Commons Attribution Non Commercial (CC BY-NC 4.0) license, which 
permits others to distribute, remix, adapt, build upon this work non-commercially, and license their derivative works on different terms, provided the original work is properly cited and the use is non-commercial. See: http://creativecommons.org/ licenses/by-nc/4.0/

(C) Article author(s) (or their employer(s) unless otherwise stated in the text of the article) 2018. All rights reserved. No commercial use is permitted unless otherwise expressly granted.

\section{REFERENCES}

1. Esserman LJ, Thompson IM, Reid B, et al. Addressing overdiagnosis and overtreatment in cancer: a prescription for change. Lancet Oncol 2014;15:e234-42.

2. Cancer Research UK. Cancerstats: thyroid cancer incidence statistics. http://www.cancerresearchuk.org/cancer-info/cancerstats/ types/thyroid/incidence/uk-thyroid-cancer-incidence-statistics (accessed 30 Aug 2017).

3. Canadian Cancer Society's Advisory Committee on Cancer Statistics. Canadian cancer statistics. 2017. http://www.cancer. $\mathrm{ca} /$ /media/cancer.ca/CW/publications/Canadian\%20Cancer\% 20Statistics/Canadian-Cancer-Statistics-2017-EN.pdf (accessed 30 Aug 2017).

4. American Cancer Society: cancer facts and figures 2017. https:// www.cancer.org/content/dam/cancer-org/research/cancer-facts-andstatistics/annual-cancer-facts-and-figures/2017/cancer-facts-andfigures-2017.pdf (accessed 30 Aug 2017).

5. EUCAN (International Agency for Research on Cancer of the World Health Organization). Cancer fact sheets: thyroid cancer - both sexes. http://eco.iarc.fr/eucan/Cancer.aspx?Cancer=35 (accessed 30 Aug 2017).

6. Guay B, Johnson-Obaseki S, McDonald JT, et al. Incidence of differentiated thyroid cancer by socioeconomic status and urban residence: Canada 1991-2006. Thyroid 2014;24:552-5.

7. Kent WD, Hall SF, Isotalo PA, et al. Increased incidence of differentiated thyroid carcinoma and detection of subclinical disease. CMAJ 2007;177:1357-61.

8. Pathak KA, Leslie WD, Klonisch TC, et al. The changing face of thyroid cancer in a population-based cohort. Cancer Med 2013;2:537-44.

9. Davies L, Welch HG. Current thyroid cancer trends in the United States. JAMA Otolaryngol Head Neck Surg 2014;140:317-22.

10. Albores-Saavedra J, Henson DE, Glazer E, et al. Changing patterns in the incidence and survival of thyroid cancer with follicular phenotype--papillary, follicular, and anaplastic: a morphological and epidemiological study. Endocr Pathol 2007;18:1-7.

11. Enewold L, Zhu K, Ron E, et al. Rising thyroid cancer incidence in the united states by demographic and tumor characteristics, 1980-2005. Cancer Epidemiology Biomarkers \& Prevention 2009;18:784-91.

12. Chen AY, Jemal A, Ward EM. Increasing incidence of differentiated thyroid cancer in the United States, 1988-2005. Cancer 2009;115:3801-7.

13. Haugen BR, Alexander EK, Bible KC, et al. 2015 American Thyroid Association management guidelines for adult patients with thyroid nodules and differentiated thyroid cancer: the american thyroid association guidelines task force on thyroid nodules and differentiated thyroid cancer. Thyroid 2016;26:1-133.

14. Perros P, Boelaert K, Colley S, et al. Guidelines for the management of thyroid cancer. Clin Endocrinol 2014;81(Suppl 2):1-122.

15. Pacini F, Castagna MG, Brilli L, et al. Thyroid cancer: ESMO clinical practice guidelines for diagnosis, treatment and follow-up. Ann Oncol 2012;23(suppl 7):vii110-19.
16. Leboulleux S, Tuttle RM, Pacini F, et al. Papillary thyroid microcarcinoma: time to shift from surgery to active surveillance? Lancet Diabetes Endocrinol 2016;4:933-42.

17. Brito JP, Hay ID, Morris JC. Low risk papillary thyroid cancer. BMJ 2014;348:g3045.

18. Haymart MR, Miller DC, Hawley ST. Active surveillance for lowrisk cancers - a viable solution to overtreatment? N Engl J Med Overseas Ed 2017;377:203-6.

19. Sugitani I, Toda K, Yamada K, et al. Three distinctly different kinds of papillary thyroid microcarcinoma should be recognized: our treatment strategies and outcomes. World J Surg 2010;34:1222-31.

20. Ito $Y$, Miyauchi A, Kihara M, et al. Patient age is significantly related to the progression of papillary microcarcinoma of the thyroid under observation. Thyroid 2014;24:27-34.

21. Tuttle RM, Fagin JA, Minkowitz G, et al. Natural history and tumor volume kinetics of papillary thyroid cancers during active surveillance. JAMA Otolaryngol Head Neck Surg 2017;143:1015.

22. Oda $\mathrm{H}$, Miyauchi $A$, Ito $\mathrm{Y}$, et al. Incidences of unfavorable events in the management of low-risk papillary microcarcinoma of the thyroid by active surveillance versus immediate surgery. Thyroid 2016;26:150-5

23. D'Agostino TA, Shuk E, Maloney EK, et al. Treatment decision making in early-stage papillary thyroid cancer. Psychooncology 2018;27:61-8.

24. Carver CS. You want to measure coping but your protocol's too long: consider the brief COPE. Int J Behav Med 1997;4:92-100.

25. Herschbach $P$, Berg P, Dankert A, et al. Fear of progression in chronic diseases: psychometric properties of the Fear of Progression Questionnaire. J Psychosom Res 2005;58:505-11.

26. Mehnert A, Herschbach P, Berg P, et al. [Fear of progression in breast cancer patients--validation of the short form of the Fear of Progression Questionnaire (FoP-Q-SF)]. Z Psychosom Med Psychother 2006;52:274-88.

27. Theunissen M, Peters ML, Schouten EG, et al. Validation of the surgical fear questionnaire in adult patients waiting for elective surgery. PLoS One 2014;9:e100225.

28. Bunn $\mathrm{H}, \mathrm{O}^{\prime}$ Connor $\mathrm{A}$. Validation of client decision-making instruments in the context of psychiatry. Can J Nurs Res 1996;28:13-27.

29. Holmes-Rovner M, Kroll J, Schmitt N, et al. Patient satisfaction with health care decisions: the satisfaction with decision scale. Med Decis Making 1996;16:58-64.

30. Brehaut JC, O'Connor AM, Wood TJ, et al. Feldman-Stewart D: validation of a decision regret scale. Medical Decision Making 2003;23:281-92.

31. Zigmond AS, Snaith RP. The hospital anxiety and depression scale. Acta Psychiatr Scand 1983;67:361-70.

32. Gning I, Trask PC, Mendoza TR, et al. Development and initial validation of the thyroid cancer module of the M. D. Anderson Symptom Inventory. Oncology 2009;76:59-68.

33. Hopwood P, Fletcher I, Lee A, et al. A body image scale for use with cancer patients. Eur J Cancer 2001;37:189-97.

34. Norman GR, Sloan JA, Wyrwich KW. Interpretation of changes in health-related quality of life: the remarkable universality of half a standard deviation. Med Care 2003;41:582-92.

35. Castro FG, Kellison JG, Boyd SJ, et al. A methodology for conducting integrative mixed methods research and data analyses. $J$ Mix Methods Res 2010;4:342-60.

36. Sawka AM, Rilkoff $H$, Tsang RW, et al. The rationale of patients with early-stage papillary thyroid cancer for accepting or rejecting radioactive iodine remnant ablation. Thyroid 2013;23:246-7. 